\title{
Characterizing the Diversity in Users' Perceptions
}

\author{
Evangelos Karapanos and Jean-Bernard Martens \\ Department of Industrial Design \\ Eindhoven University of Technology \\ P.O. Box 513, 5600 MB, Eindhoven, The Netherlands \\ \{E.Karapanos, J.B.O.S.Martens\} atue.nl
}

\begin{abstract}
This paper proposes a novel approach to modeling the diversity in users' perceptions, based on a mixture of qualitative and quantitative techniques: the Repertory Grid Technique and Multi-Dimensional Scaling. The proposed method can be used for identifying diverse user groups that can inspire a range of personas, or for selecting subjects for field studies and usability tests. In a case study we explored the perceptions of product creators and end users towards an innovative product in its early design stage.
\end{abstract}

Keywords: user profiling, Repertory Grid, Multi-dimensional Scaling.

\section{Introduction}

Understanding the diversity in users has been one of the core challenges in usercentered design. User profiling is an essential first step towards identifying the right personas for design [3], or the right subjects for field studies and usability tests [4]. Yet, current approaches to user profiling distinguish users in terms of knowledge, educational or social background, age and gender. Such information is probably overly generic and does not reflect how users differ in terms of their attitude towards a specific product under development. At the same time there is growing evidence that designers underestimate the diversity in users [4]. As a result, developed personas may lack a connection to important target users [3, 6], and subjects in user studies may not represent the wide range of actual (or potential) users of a product [4]. The need for a closer link between user profiling and product design has been repeatedly highlighted [1,3], but as yet, novel approaches are rather scarce (e.g. [6]).

This paper describes a new method for understanding the diversity in users perceptions' and identifying homogeneous user groups, based on a mixture of qualitative and quantitative techniques. The method consists of three stages. First, users' idiosyncratic views are elicited in a structured interview approach using the Repertory Grid Technique (RGT) [2]. Second, a user segmentation map that expresses the diversity among users is derived from their dissimilarity ratings by means of Multi-Dimensional Scaling (MDS) [5]. Homogeneous groups of users are identified within this map by means of (hierarchical) clustering. Third, perceptual maps are created from the attribute, dissimilarity and preference ratings to express how homogeneous groups of subjects perceive the products being studied. 
We have applied the new approach in a case study where we explored the perceptions of six related products by a diverse group of subjects, consisting of product creators and end users. One of the goals of the study was to identify discrepancies (i) between product creators' and end-users' perceptions, and (ii) between different individuals within a multi-disciplinary concept design team. In the following sections we describe the proposed method for user profiling, and illustrate it with concrete results from our case study.

\section{Case Study}

Eleven persons directly involved in the concept design phase of a new product, and eleven potential end users, participated in the case study. The end users were researchers from our department who had no prior knowledge of the product under development. The product creators were all employees of the R\&D department of an international company developing document systems. They were all involved in the conception and realization of TouchToPrint, which is a new way of personalizing the use of a shared printer by means of fingerprint recognition. This new concept and five alternative proposals for interacting with a shared printer were presented to the participants in the form of posters. Each poster described a usage scenario of the relevant concept. First, the six products were combined in three triads. For every triad, participants were asked to "think of a property or quality that makes two of the products alike and discriminates them from the third". This resulted in a list of attributes, product qualities that users perceive and use while forming overall evaluations of a product. Afterwards, the subjects were asked to rate all products on their personal attributes, as well as on preference and dissimilarity. In contrast to the traditional Repertory Grid approach, we employed paired comparisons instead of semantic differentials, as this was a priori expected to deliver more stable results [5].

\section{Analysis}

The analysis process consists of two steps. First, a user segmentation map is created for identifying homogeneous groups in the participant sample. Second, a perceptual map is created for each homogeneous group to gain insight into how products are perceived.

To create the user segmentation map, we define the distance $D_{i j}=1-R^{2}$ ij between participants $i$ and $j$ based on the correlation $R_{i j}$ between their dissimilarity scores. Derived distances are then visualized in two or more dimensions (Fig. 1) using the MDS tool XGms [5]. Hierarchical clustering (with minimum variance) reveals two main clusters that can be further subdivided into five more or less homogeneous participant groups. Groups 3 and 4 consist entirely of end users, while groups 1, 2 and 5 consist mostly of product creators. Identification of the product creators reveals that group 1 consists mostly of technically-oriented product creators, while group 2 consists mostly of user-oriented product creators.

A perceptual space representing the products is subsequently constructed for each homogenous group, based on the dissimilarity scores from all participants within the group. We only present the results for groups 1 and 3 . Both perceptual spaces can be 


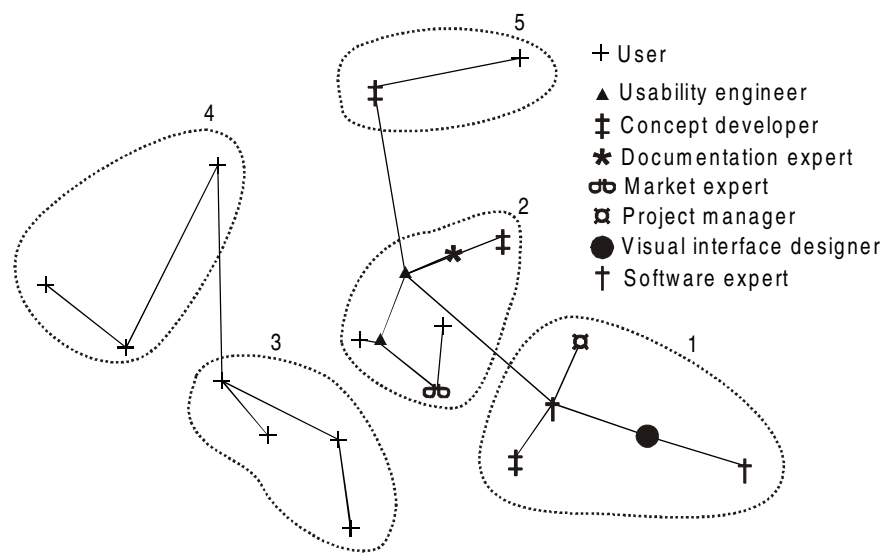

Fig. 1. User segmentation map based on correlations between dissimilarity scores

adequately visualized in two dimensions, and attribute vectors are fitted into these spaces by performing multiple regressions between the stimulus configuration (as independent variables) and the attribute scores (as the dependent variables). Only significant attributes are retained in the visualizations of Fig. 2.
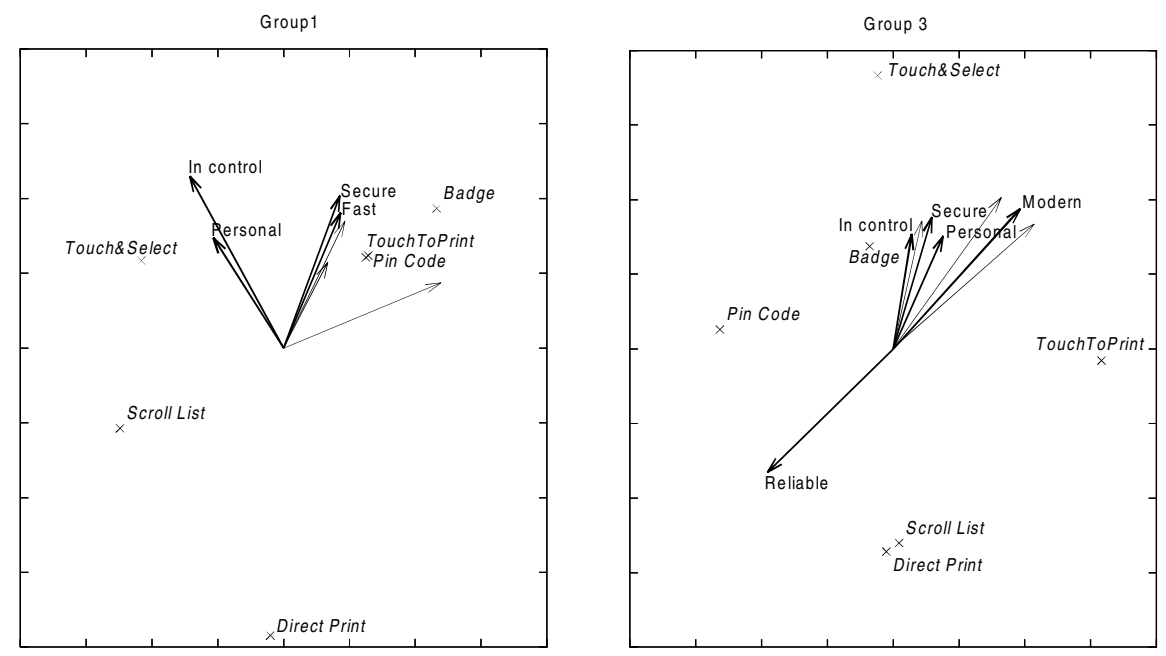

Fig. 2. Perceptual maps for groups 1 and 3. The light vectors represent individual preferences, while the other vectors represent the most significant attributes.

For individuals in group 1, secure and fast are the most important product qualities influencing their preference judgment, while participants in group 3 value modern, personal and secure products. Subjects within group 3 have negative concerns regarding the reliability of new products, such as Touch\&Select, TouchToPrint and Badge, despite the fact that they prefer them. Such concerns are not reported by the subjects in group 1. 


\section{Conclusion}

This paper has presented a new experimental approach to understanding the diversity in product perceptions between people. The proposed method has several advantages. First, it follows a pragmatic approach to user modeling by distinguishing users based on how they perceive and appreciate products rather than on abstract demographic and psychographic information. Second, it also accounts for users' idiosyncratic views in contrast to other approaches [6] where a priori defined attributes are imposed in the evaluation. Third, the method can be used in different phases of the product development, for instance using concept sketches, as was done in our case study, but also for benchmarking between competitor products. Fourth, the method consists of two distinct stages that can be handled in a flexible way. Only stage one, i.e., the elicitation of attributes using the RGT, requires a more experienced experimenter and is therefore practically restricted to a limited number of subjects. Stage two that consists of rating the collected attributes, as well as preferences and dissimilarities, can easily be automated and conducted with a large number of subjects.

In the future, we want to compare the proposed method of user profiling with alternative methods based on market analysis or logging of actual product use. More specifically, within an existing project we are interested in a better understanding of the relationship between user profiles and customer complaints. We also want to establish more closely how useful the technique is in informing the construction of personas. Personas derived from this method will be compared to others derived from traditional user segmentation techniques in terms of how useful they are in the followup design process.

Acknowledgments. We thank Fred de Jong from Océ Technologies for his help throughout the study, and all participants for their valuable input. This work is being carried out as part of the "Soft Reliability" project, sponsored by the Dutch Ministry of Economic Affairs under the IOP-IPCR program.

\section{References}

1. Dillon, A., Watson, C.: User analysis in HCI - The historical lessons from individual differences research. International Journal of Human-Computer Studies , 619-637 (1996)

2. Fransella, F., Bell, R., Bannister, D.: A Manual for Repertory Grid Technique. Wiley, Chichester (2003)

3. Grudin, J., Pruitt, J.: Personas, participatory design and product development: An infrastructure for engagement. In: Proceedings of Participatory Design Conference, Palo Alto, pp. 144-161 (2002)

4. Kujala, S., Kauppinen, M.: Identifying and selecting users for user-centered design. In: Proceedings of the third Nordic conference on Human-computer interaction, ACM Press, Tampere, Finland (2004)

5. Martens, J.-B.: Image technology design: A perceptual approach. Kluwer Academic Publisher, Boston (2003)

6. Sinha, R.: Persona development for information-rich domains. In: CHI '03 extended abstracts on Human factors in computing systems, ACM Press, Florida, USA (2003) 